\begin{tabular}{ll|l} 
Case Reports in & \multicolumn{2}{c}{ Case Rep Gastroenterol 2013;7:470-475 } \\
\cline { 2 - 3 } Gastroenterology & $\begin{array}{l}\text { DOI: 10.1159/000356817 } \\
\text { Publisned online: November 6, 2013 }\end{array}$ & $\begin{array}{l}\text { ○ 2013 S. Karger AG, Basel } \\
\text { www.karger.com/crg }\end{array}$ \\
\hline & $\begin{array}{l}\text { This is an Open Access article licensed under the terms of the Creative Commons } \\
\text { Attribution-NonCommercial 3.0 Unported license (CC BY-NC) (www.karger.com/OA- } \\
\text { license), applicable to the online version of the article only. Distribution permitted for non- } \\
\text { commercial purposes only. }\end{array}$
\end{tabular}

\title{
Life-Threatening Gastrointestinal Mucosal Necrosis during Methotrexate Treatment for Rheumatoid Arthritis
}

\author{
Tomoya Tsukada ${ }^{a, b}$ Tatsuo Nakano ${ }^{b}$ Takashi Miyata ${ }^{a, b}$ Shozo Sasaki ${ }^{b}$ \\ ${ }^{a}$ Department of Gastroenterologic Surgery, Kanazawa University, and ${ }^{b}$ Department of \\ Surgery, Asanogawa General Hospital, Kanazawa, Japan
}

\section{Key Words}

Methotrexate - Adverse effect - Drug lymphocyte stimulation test - Type IV allergic reactions . Gastrointestinal tract $\cdot$ Mucosal injury $\cdot$ Necrosis $\cdot$ Rheumatoid arthritis

\begin{abstract}
Methotrexate (MTX), a folic acid antagonist, is widely used in the treatment of neoplasms, psoriasis and rheumatoid arthritis. Despite its efficacy, MTX sometimes finds limited application because of its adverse effects, including renal or liver impairment, bone marrow toxicity and gastrointestinal mucosal injury. Intestinal mucositis, bleeding and peptic ulcers are well-known gastrointestinal adverse effects of MTX, although cases of fatal mucosal necrosis induced by MTX are extremely rare. Here, we report the case of an 82-year-old Japanese woman who developed severe gastrointestinal mucosal necrosis after 8 years of treatment with low-dose MTX ( $8 \mathrm{mg} /$ week). In the drug lymphocyte stimulation test, MTX showed a strong positive reaction, with a stimulation index of $443 \%$ against normal controls. Physicians must be aware of potential drug-induced adverse effects in patients with chronic diseases who are on long-term medication.
\end{abstract}

\section{Introduction}

Methotrexate (MTX) is a well-known immunosuppressive agent classified under disease-modifying anti-rheumatic drugs (DMARDs), and it is widely used in the treatment of neoplasms, psoriasis and rheumatoid arthritis (RA). Several controlled studies have demonstrated the usefulness of low-dose intermittent MTX treatment in RA patients, and MTX is used as the first-line agent because of its limited toxicity $[1,2]$. The adverse effects of MTX, such as renal or liver impairment, bone marrow toxicity and gastrointestinal mucosal injury,

Tomoya Tsukada

Department of Gastroenterologic Surgery, Kanazawa University

13-1 Takara-machi, Kanazawa, Ishikawa 920-8641 (Japan)

E-Mail tkd_tmy@nifty.com 
Tsukada et al.: Life-Threatening Gastrointestinal Mucosal Necrosis during Methotrexate Treatment for Rheumatoid Arthritis

may be related to peak dosage, and many of them can often be avoided by dose reduction or combined use of MTX and folic acid [3]. However, even low-dose MTX can cause severe adverse effects such as acute pneumonitis [4] and leukoencephalopathy [5]. Intestinal mucositis, bleeding and peptic ulcers are other well-known gastrointestinal adverse effects of MTX, although fatal mucosal necrosis induced by MTX is extremely rare. Here, we report a case of severe gastrointestinal necrosis induced by long-term low-dose MTX treatment. This condition was diagnosed on the basis of the clinical course and laboratory data, including the results of the drug lymphocyte stimulation test (DLST).

\section{Case Report}

An 82-year-old woman was referred to our hospital for constipation and lower abdominal pain 3 days after she had taken her last dose of MTX. She had been diagnosed with RA at the age of 74 and was being treated with nonsteroidal anti-inflammatory drugs (NSAIDs; $180 \mathrm{mg} /$ day loxoprofen sodium hydrate), prednisolone (4-6 mg/day) and lowdose MTX (8 mg/week). At the first visit to our hospital, physical examination showed fullness and slight tenderness of the lower abdomen. Blood tests showed that she had anemia (hemoglobin [Hb] $10.0 \mathrm{~g} / \mathrm{dl}$ ) and mild inflammation (white blood cell [WBC] count and C-reactive protein [CRP] levels were $18,600 / \mu \mathrm{l}$ and $0.95 \mathrm{mg} / \mathrm{dl}$, respectively). Her platelet count was $388,000 / \mu \mathrm{l}$, and biochemical examination showed no significant abnormality. Computed tomography of the abdomen showed slight thickening of the descending colonic wall and no organic lesions resembling neoplasms. Lower gastrointestinal endoscopy showed mucosal petechiae in the descending colon, and the patient was believed to have colitis caused by fecal impaction (fig. 1). Her symptoms were immediately relieved once she defecated after receiving laxative treatment. Antibiotics were not administered. To provide bowel rest, ingestion of enteral nutrients and parenteral nutrition were started.

Nine days after the first visit, the patient again complained of nausea, epigastralgia and abdominal fullness. The next day, she developed a fever $\left(38.6^{\circ} \mathrm{C}\right)$ and ulcerative oral mucositis. Laboratory examination showed a WBC count of $900 / \mu \mathrm{l}$, a $\mathrm{Hb}$ level of $7.6 \mathrm{~g} / \mathrm{dl}$, a platelet count of $120,000 / \mu \mathrm{l}$ and a CRP level of $14.45 \mathrm{mg} / \mathrm{dl}$. Upper gastrointestinal endoscopy showed severe mucosal necrosis covering a large area (almost the entire circumference) of the lower esophagus, linear erosive gastritis and multiple duodenal ulcers. Lower gastrointestinal endoscopy was performed again, and mucosal necrosis with sloughing was observed along the entire circumference of the descending colon (fig. 2a-d). Contrastenhanced computed tomography showed wall thickening along the entire circumference of the lower esophagus and descending colon, with mild pleural effusion. Microscopic examination of biopsy specimens from the esophagus and descending colon also revealed necrotic mucosa. On fecal culture analysis conducted at the same time, methicillin-resistant Staphylococcus aureus (MRSA) was detected, but Clostridium difficile or Candida albicans was not. Therapy with granulocyte colony-stimulating factor and empiric antibiotics was initiated. Eleven days after the first visit, the patient developed pancytopenia; her WBC count was $400 / \mu \mathrm{l}, \mathrm{Hb}$ was $6.1 \mathrm{~g} / \mathrm{dl}$ and platelet count was $59,000 / \mu \mathrm{l}$. Severe inflammation and renal injury were observed (CRP $27.99 \mathrm{mg} / \mathrm{dl}$; blood urea nitrogen $84 \mathrm{mg} / \mathrm{dl}$; creatinine level $1.18 \mathrm{mg} / \mathrm{dl}$ ). Blood transfusion and intravenous administration of meropenem, vancomycin, gamma-globulin, recombinant human soluble thrombomodulin and a proton pump inhibitor were started for the pancytopenia and infection. Leucovorin rescue was also initiated with a 3-mg intravenous bolus every $8 \mathrm{~h}$, since the involvement of MTX was suspected. However, since the patient's general condition did not improve immediately, continuous hemodiafil- 
Tsukada et al.: Life-Threatening Gastrointestinal Mucosal Necrosis during Methotrexate Treatment for Rheumatoid Arthritis

tration with polymyxin B-immobilized fibers was performed. After 2 days of this blood purification therapy, the patient's general condition improved gradually.

The DLST was performed for MTX, NSAIDs and prednisolone. MTX showed a strong positive reaction, with a stimulation index of $443 \%$ compared to drug-free normal controls. The patient resumed oral intake 30 days after her first visit but had developed cicatricial stricture of the esophagus and colon (fig. 2e-h). Therefore she required balloon dilatation, twice for the esophagus and once for the descending colon. She was discharged from our hospital 15 weeks after admission (fig. 3).

\section{Conclusions}

Gastrointestinal mucosal injuries (i.e. erosions, ulcers and perforations) caused by steroids, NSAIDs and DMARDs are common in RA patients. Although perforations are typical severe gastrointestinal complications, Curtis et al. [6] reported that the frequency of perforations is about $0.1 \%$ in RA patients. The use of MTX, especially low-dose MTX, is not an independent risk factor of intestinal perforation, and it is considered a relatively safe treatment. However, fatal adverse effects of MTX, such as toxic epidermal necrolysis [7], bone marrow aplasia [8] and pneumonia [9] have been reported. With regard to the gastrointestinal tract, it has been reported that even at low doses of $5 \mathrm{mg} / \mathrm{m}^{2}$, MTX can cause immunosuppressive effects that can result in life-threatening complications due to bacterial infection [10].

In the present case, the added complication of MRSA enteritis was considered to have resulted in the severe clinical course. However, the entire gastrointestinal mucosal lesion cannot be explained only by the presence of MRSA enteritis. It is conceivable that the infection caused systemic inflammatory syndrome or thrombotic microangiopathy, but the cause is unlikely to be a simple pathophysiological one, since multiple organ injury developed concurrently. Therefore, it is speculated that MTX-induced gastrointestinal mucositis develops on the basis of patients' background. The pathogenic mechanism underlying this mucositis remains unknown, but immune response and increased blood concentration could be considered as the causes. Chronic constipation in older women causes the retention of parenteral medication in the gastrointestinal tract. The blood concentration may increase rapidly if the absorption capacity is improved by lack of defecation and fasting. In addition, long-term use of drugs may induce allergic reactions and cause mucosal damage.

The DLST is an in vitro test used to identify type IV allergic reactions to drugs; thus, it is both safe and useful as an accessory diagnostic tool [11]. In the present case, it was not possible to measure the serum concentration of MTX. However, the lymphocyte proliferation test confirmed an immune response to MTX, considering the patient's clinical course.

To our knowledge, no studies have examined the results of the DLST against severe gastrointestinal mucosal injury caused by MTX. Management of the present case was successful in that the patient was treated after rapid identification of the drug involved using the DLST and administration of intensive care.

Physicians should be aware of potential drug-induced adverse effects in patients with chronic diseases who are on long-term medication. Additionally, further studies are needed on the safe use of MTX and the pathogenic mechanism underlying the drug-induced immune response. 
Tsukada et al.: Life-Threatening Gastrointestinal Mucosal Necrosis during Methotrexate Treatment for Rheumatoid Arthritis

\section{Author Contributions}

T. Tsukada participated in treatment of the patient, collected case details, conducted a literature search and drafted the manuscript. T. Nakano participated in treatment of the patient and helped draft the manuscript. T. Miyata and S. Sasaki participated in treatment of the patient. All authors read and approved the final manuscript.

\section{Disclosure Statement}

The authors declare that they have no conflicts of interest or competing interests.

\section{References}

1 Bologna C, Viu P, Picot MC, et al: Long-term follow-up of 453 rheumatoid arthritis patients treated with methotrexate: an open, retrospective, observational study. Br J Rheumatol 1997;36:535-540.

-2 Kremer JM, Russell AS, Emery P, et al: Long-term safety, efficacy and inhibition of radiographic progression with abatacept treatment in patients with rheumatoid arthritis and an inadequate response to methotrexate: 3-year results from the AIM trial. Ann Rheum Dis 2011;70:1826-1830.

-3 Hoekstra M, van Ede AE, Haagsma CJ, et al: Factors associated with toxicity, final dose, and efficacy of methotrexate in patients with rheumatoid arthritis. Ann Rheum Dis 2003;62:423-426.

4 Hargreaves MR, Mowat AG, Benson MK: Acute pneumonitis associated with low dose methotrexate treatment for rheumatoid arthritis: report of five cases and review of published reports. Thorax 1992;47: 628-633.

5 Matsuda M, Kishida D, Kinoshita T, et al: Leukoencephalopathy induced by low-dose methotrexate in a patient with rheumatoid arthritis. Intern Med 2011;50:2219-2222.

-6 Curtis JR, Xie F, Chen L, et al: The incidence of gastrointestinal perforations among rheumatoid arthritis patients. Arthritis Rheum 2011;63:346-351.

7 Primka EJ 3rd, Camisa C: Methotrexate-induced toxic epidermal necrolysis in a patient with psoriasis. J Am Acad Dermatol 1997;36:815-818.

-8 Copur S, Dahut W, Chu E, et al: Bone marrow aplasia and severe skin rash after a single low dose of methotrexate. Anticancer Drugs 1995;6:154-157.

-9 Ridley MG, Wolfe CS, Mathews JA: Life threatening acute pneumonitis during low dose methotrexate treatment for rheumatoid arthritis: a case report and review of the literature. Ann Rheum Dis 1988;47: 784-788.

10 Nanke Y, Kotake S, Akama H, et al: Pancytopenia and colitis with Clostridium difficile in a rheumatoid arthritis patient taking methotrexate, antibiotics and non-steroidal anti-inflammatory drugs. Clin Rheumatol 2001;20:73-75.

11 Arai Y, Arihiro S, Ide D, et al: Acute pancreatitis due to $\mathrm{pH}$-dependent mesalazine that occurred in the course of ulcerative colitis. Case Rep Gastroenterol 2011;5:610-616. 
Tsukada et al.: Life-Threatening Gastrointestinal Mucosal Necrosis during Methotrexate Treatment for Rheumatoid Arthritis
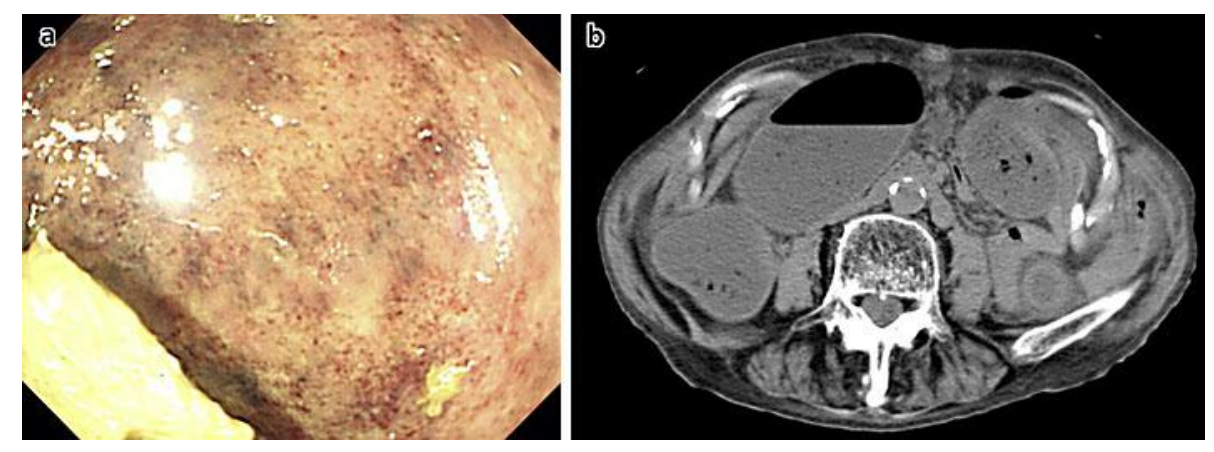

Fig. 1. Images recorded at the initial visit. a Lower gastrointestinal endoscopy showing mucosal petechiae in the descending colon. b Computed tomography of the abdomen showing slight thickening of the descending colonic wall.

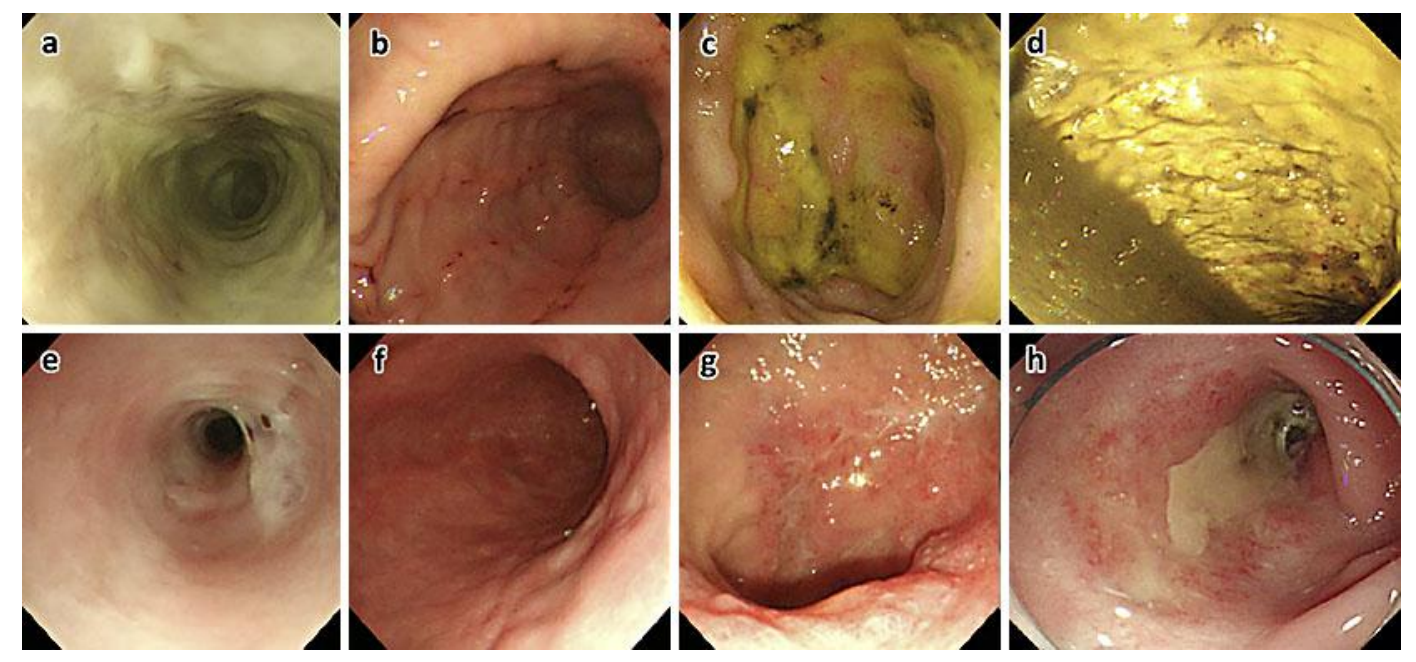

Fig. 2. Pre-therapy and follow-up endoscopic images. Pre-therapy images: a Esophagus. Necrosis of the entire mucosa in the lower esophagus. b Stomach. Linear erosions from the antrum to the body. c Duodenum. Multiple duodenal ulcers in the bulbus. d Descending colon. Necrosis of the entire mucosa with sloughing. Follow-up images: e Esophagus. Mucosal necrosis is improved, but the stenosis remains. f Stomach. No signs of mucosal injury. g Duodenum. Ulcers tending to heal. h Descending colon. Mucosal necrosis is improved, but the stenosis remains. 
Tsukada et al:: Life-Threatening Gastrointestinal Mucosal Necrosis during Methotrexate Treatment for Rheumatoid Arthritis

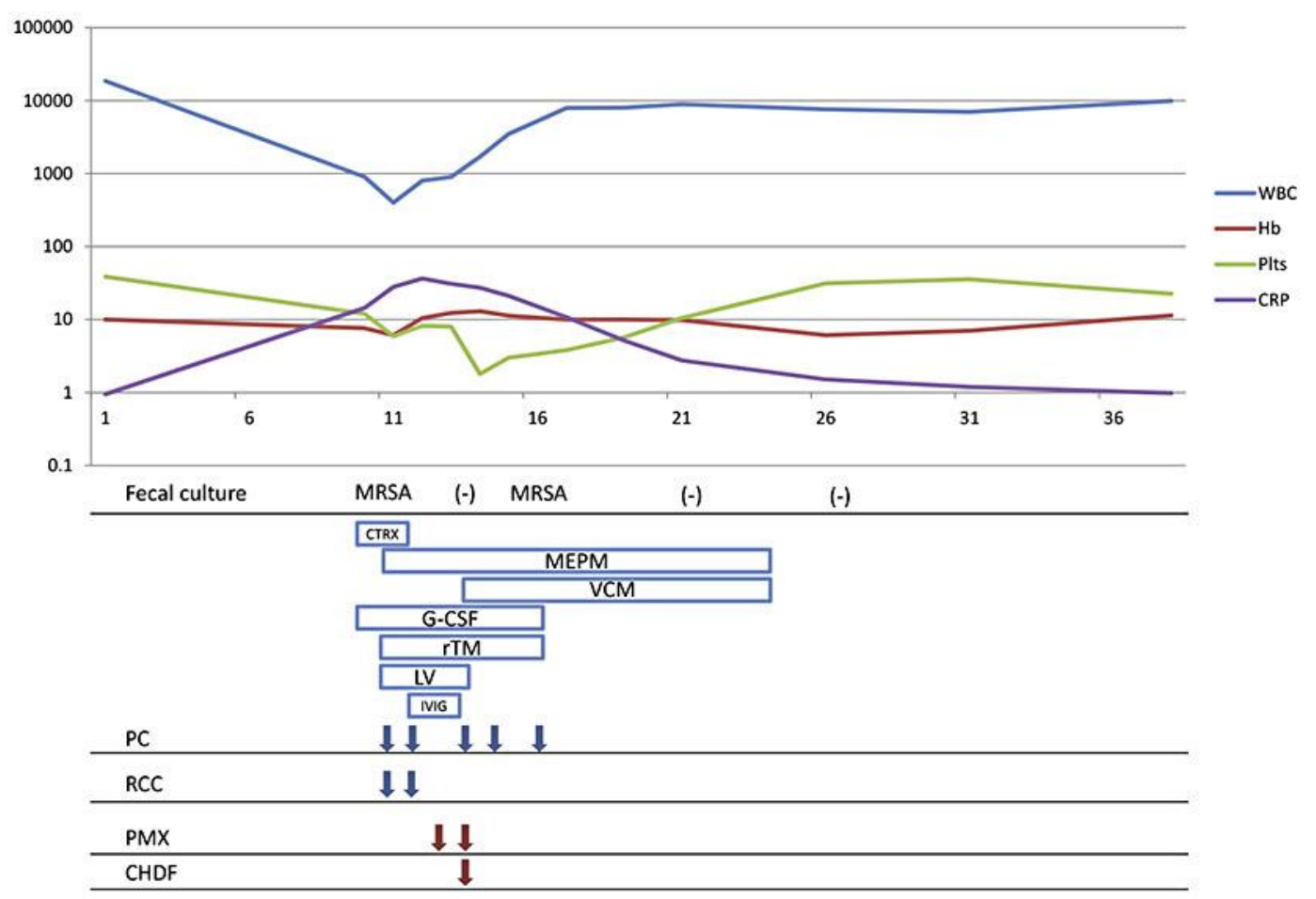

Fig. 3. Clinical course of the patient. CTRX = Ceftriaxone; $\mathrm{MEPM}=$ meropenem; $\mathrm{VCM}=$ vancomycin; G-CSF = granulocyte colony-stimulating factor; rTM = recombinant human soluble thrombomodulin; $\mathrm{LV}=$ leucovorin; IVIG = intravenous immunoglobulin; $\mathrm{PC}=$ platelet concentrate; $\mathrm{RCC}=$ red cell concentrate; $\mathrm{PMX}=$ polymyxin B-immobilized fibers; $\mathrm{CHDF}=$ continuous hemodiafiltration. 